\title{
单个纳米颗粒的上转换光谱现象研究
}

\author{
周佳佳，邱建荣 \\ (浙江大学 光电科学与工程学院, 现代光学仪器国家重点实验室, 杭州 310027)
}

摘 要: 稀土离子掺杂上转换纳米颗粒具有独特的光谱学特征, 在太阳能利用、三维显示和生物医学等众多领域有 着广泛的应用价值。然而, 传统基于统计平均效应的上转换纳米颗粒群体性光谱学研究忽视了众多来自单个颗粒的 非均一性信息。单颗粒光谱检测成为一种突破上述瓶颈，获取源自纳米颗粒非均一性本征结构的独特光谱现象，并 实现单个纳米颗粒器件化的有效技术手段。对于单个纳米颗粒电子行为的研究能够揭示来自微结构本身的信息，甚 至能够在没有介质干扰下清晰洞见材料本征结构和外部作用的相互影响，从而为制备高质量的纳米颗粒提供指导 意义。同时, 单颗粒光谱检测也具有在微纳米尺度探索晶体结构各向异性光学特性及一些尚未预见的新型光学现象 的强大能力。本文介绍了单个纳米颗粒上转换发光表征的重要性以及常见的几种检测方法。论述了单颗粒上转换 发光的一些最新研究成果, 并对未来发展方向进行了展望。

关 键 词: 上转换发光; 稀土离子; 单颗粒; 光谱学; 综述

中图分类号: TQ174 文献标识码: A

\section{Upconversion Spectroscopic Investigation of Single Nanoparticles}

\section{ZHOU Jia-Jia, QIU Jian-Rong}

(College of Optical Science and Engineering, State Key Laboratory of Modern Optical Instrumentation, Zhejiang University, Hangzhou 310027, China)

\begin{abstract}
Upconversion nanoparticles (UCNPs) have made a significant and valuable contribution to photophysics and biomedicine, due to their specific spectroscopic characteristics. However, the ensemble spectroscopy of UCNPs is limited for the electronic behavior in average effect, which ignores the fact that the nanoparticles are heterogeneous. Towards the research focus on heterogeneous intrinsic structure, unique photophysical phenomena, and advanced applications, the optical characterization of single UCNPs are promoted to a frontier breakthrough of UCNPs community. Electronic behavior detection aimed at a single nanoparticle displays signals from the micro-structure of nanoparticles, while single nanoparticle spectroscopy offers clear insight into the interplay between intrinsic and extrinsic influences without noise, and subsequently gives instructions for the high quality preparation of UCNPs. Single nanoparticle optical characterization possesses a powerful capacity to explore the crystalline structure anisotropy related optical differences, or some unexpected unique optical phenomena at sub-micron and even nano-scale. In this review, the importance of single UCNPs characterization and single particle detection methods are overviewed, in which the considerable emphasis is placed on the specific spectroscopic study of single UCNPs Showing fantastic photophysical phenomena beyond ensemble measurement. Finally, this review identifies
\end{abstract}

收稿日期: 2016-03-08; 收到修改稿日期：2016-04-14

基金项目：国家自然科学基金青年项目(11404311)；浙江省自然科学基金(LY14E020007) National Natural Science Foundation of China (11404311); Zhejiang Provincial Natural Science Foundation of China (LY14E020007)

作者简介：周佳佳(1985-), 女，博士，讲师. E-mail: zhoujiajia@zju.edu.cn

通讯作者: 邱建荣, 教授. E-mail: qjr@zju.edu.cn 
promising opportunities in which single UCNPs characterization technique can accelerate on-going research with a remarkable depth and breadth, facilitate discovery of upconversion nanoparticles that overcome fundamental limitations of current ensemble level.

Key words: upconversion; lanthanides; single nanoparticle; spectroscopy; review

稀土离子掺杂上转换发光是一个依赖于稀土离 子丰富的能级特征, 通过吸收两个或多个低能光子 转变成一个高能光子的光致发光过程。早在 20 世纪 中期, 实现上转换发光的典型稀土离子组合及其上 转换发光物理机制已被广泛研究 ${ }^{[1]}$ 。近年来, 以 $\mathrm{NaReF}_{4}(\mathrm{Re}$ : 稀土元素)系列上转换纳米材料的可控 制备为代表, 纳米技术的迅速发展进一步将上转换 发光研究推向新的高潮 ${ }^{[2-6]}$ 。稀土离子掺杂纳米颗粒 以其独特的上转换光谱特性在生物医学、传感、三 维显示、光伏等众多领域获得了应用 ${ }^{[3,7-14]}$ 。但是不 难发现, 大多数上转换发光特性及应用的研究都集 中在纳米颗粒的群体性光学效应, 而很少关注单个 纳米颗粒的光学行为。

事实上，看似相同的粒子实际上具有不同的特 性, 而这些特性对于材料宏观性能的优化, 乃至新 材料、新性能的开发和应用都具有重要的指导意义, 尤其在考虑微纳器件时, 单个纳米颗粒的性能表征 就极为重要。就上转换纳米颗粒而言, 以 $\mathrm{NaYF}_{4}$ 为 例, 它通常因在液相环境下合成使其表面富含有机 分子。这些有机分子的存在, 一方面使颗粒具有极 好的单分散性, 并能通过表面修饰实现特定的功能 性, 另一方面从光物理学角度, 有机分子将影响稀 土离子的电子跃迁, 使上转换发光过程复杂化。颗 粒间个体的精细差异在群体性光学检测中是无法分 辨的, 单颗粒光谱研究将有望揭示微观尺度的物理 机制。

除了机理研究以外, 单颗粒光学检测也是在微 纳尺度发现新型光学现象, 观察晶体结构相关的各 向异性光学特性的有力途径。在早期研究中, 对于 量子点、金属纳米颗粒等基于单颗粒检测的新型光 学现象的探索并不少见 ${ }^{[15-21]}$ 。而上转换纳米颗粒这 类以掺杂稀土离子为发光中心的绝缘体, 发光机制 上不同于半导体材料, 并且利用单颗粒光谱能够进 一步实现深层次高精度的应用演示, 例如应用单个 上转换纳米颗粒实现光学超分辨成像, 应用单个上 转换纳米颗粒和金属颗粒的相互作用实现光学局域 场增强等。

以下就单个纳米颗粒的上转换光谱学研究为主 题, 介绍几种常见的单颗粒光谱检测方法, 并对该 领域中近期研究成果进行归纳整理。

\section{1 单颗粒光谱研究方法}

单个纳米颗粒的上转换发光检测是同时依赖于 光学工程背景与材料科学知识的一类先进检测方法, 可以高分辨高精度地揭示材料特性。通常, 以 $980 \mathrm{~nm}$ 等半导体激光为激发光源, 选择性探测紫外、可见以 及近红外波段的荧光。常见的几种探测方法包括共 聚焦显微苂光扫描法、光纤颗粒负载法和光镊法等。

\section{1 共聚焦显微荧光扫描法}

共聚焦显微荧光探测相比于宽场显微苂光探测, 因空间滤波效应而具有更高的信噪比 ${ }^{[22-23]}$ 。如图 1 所示, 只有在焦点上的信号才能穿过小孔被探测器 接收。对单个上转换纳米颗粒的荧光探测基本步骤 包括: 1)980 $\mathrm{nm}$ 激光光源通过显微物镜聚焦到样品 表面；2)样品通过压电控制三维纳米定位系统实现 逐点扫描; 3)样品各点上转换荧光通过同一物镜、分 束器、小孔和滤光片等到达探测器 ${ }^{[13,24-31]}$ 。其中, 上 转换纳米颗粒通过溶剂稀释成特定浓度后, 分散在 载玻片等衬底上。单颗粒的定位除依赖荧光亮度和 均匀度初步判断外, 还可借助原子力显微镜和透射 电镜照片进行对照分析。

\section{2 光纤颗粒负载法}

采用中空的微结构光纤吸附纳米颗粒后进行检 测, 原理如图 2 所示 ${ }^{[9,32-34]}$ 。将 $980 \mathrm{~nm}$ 激光光源通 过显微物镜耦合到负载光纤, 光纤内部的纳米颗粒 受激产生的上转换荧光, 沿光纤反向传播, 经二向 色镜、滤光片等被探测器收集。其中, 上转换纳米 颗粒通过溶剂稀释成特定浓度, 经毛细管力作用吸 入光纤。

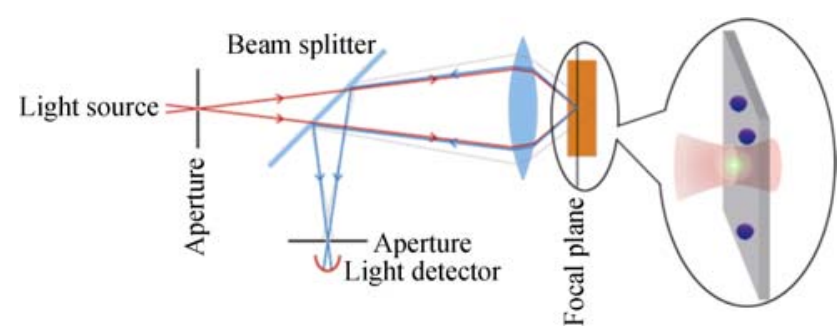

图 1 共聚焦显微荧光扫描系统原理示意图

Fig. 1 Schematic diagram of confocal microscopic fluorescent scanning system from upconversion nanoparticles 


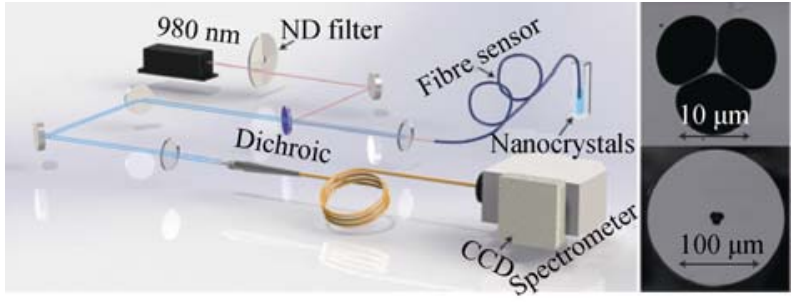

图 2 光纤颗粒负载法检测上转换发光实验系统示意图 ${ }^{[9]}$

Fig. 2 Schematic experimental configuration for capturing UC luminescence of nanoparticles using a suspended-core microstructured optical-fiber dip sensor ${ }^{[9]}$

\section{3 光镊法}

光铝法可以通过高度聚焦激光束产生的力来操 纵纳米或微米级的电介质颗粒。如图 3 所示, 在单 颗粒上转换苂光检测中, $980 \mathrm{~nm}$ 激光既用来产生力 场进行样品光学捕获, 也作为激发光源使样品产生 上转换苂光 ${ }^{[35-36]}$ 。高聚焦 $980 \mathrm{~nm}$ 激光束通过高数值 孔径显微物镜得到, 聚焦后的激光光束最窄的部分 (光束腰)存在非常强的电场梯度。上转换纳米颗粒 会被吸引至电场梯度最高的区域, 也就是光束的中 心，从而被激发，并用同一物镜收集所产生的苂光， 最终到达各探测器进行数据采集。该方法可以在液 相环境下直接对颗粒进行光学检测。

\section{2 单颗粒上转换光谱学研究}

\section{1 光稳定性}

光致闪胨在量子点、荧光素等分子发射体中是一 种常见的现象，反映了材料的光学不稳定性 ${ }^{[37-39]}$ 。对 于稀土掺杂的无机绝缘体材料，也曾有报道称在 $\mathrm{Y}_{2} \mathrm{O}_{3}$ 纳米颗粒中观察到单个 $\mathrm{Eu}^{3+}$ 呈现几百毫秒到

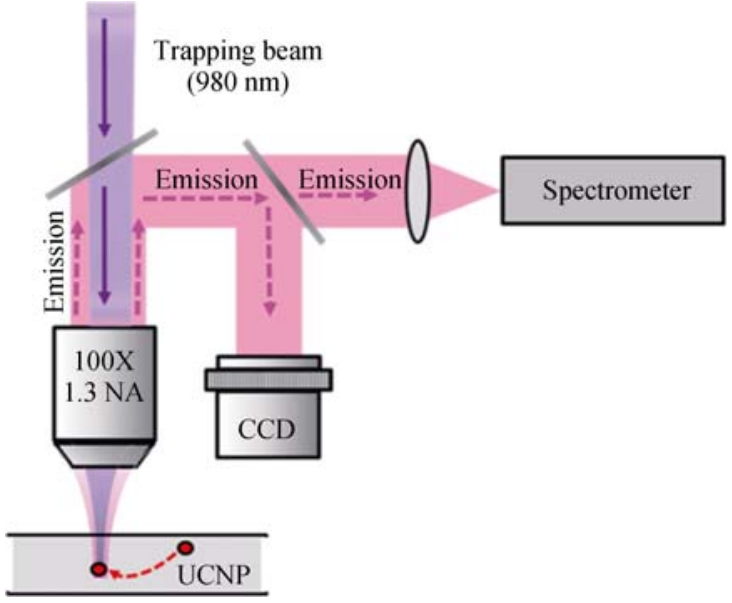

图 3 光镊法进行单颗粒上转换荧光探测系统示意图 ${ }^{[35]}$

Fig. 3 Schematic diagram of the experimental setup used for luminescence acquisition of an optically trapped upconversion nanoparticle ${ }^{[35]}$

几秒的闪胨现象 ${ }^{[40]}$ 。然而, 这一现象在富含众多稀 土离子的纳米颗粒中并不存在。对于稀土离子掺杂 的上转换纳米颗粒而言, 是否具有光学稳定性通过 单颗粒上转换苂光表征得到完美演示 ${ }^{[23-24,29] 。 ~}$ Cohen 等 ${ }^{[24]}$ 对 $\mathrm{Yb}^{3+}-\mathrm{Er}^{3+}$ 掺杂的 $\beta-\mathrm{NaYF}_{4}$ 纳米颗粒进 行长达 $1 \mathrm{~h}$ 的连续光照射, 没有观察到任何光闪胨、 光漂白或是破坏现象。至此，这种在某一时间段内 对颗粒菼光亮度波动进行观察的方法，被广泛用来 评价上转换纳米颗粒的光稳定性以及亮度比较, 如 表 1 所示。

\section{2 浓度一强度平衡机制}

稀土离子掺杂浓度是决定上转换发光强度及显 色性的一个重要决定因素。材料对光能的吸收、离 子间的能量传递以及辐射跃迁的概率都依赖于掺杂

表 1 单个上转换纳米颗粒的光稳定性和亮度评价

Table 1 Photostability and brightness assessment of single UCNPs

\begin{tabular}{|c|c|c|c|c|c|c|}
\hline UCNPs & $\begin{array}{l}\text { Particle } \\
\text { size/nm }\end{array}$ & $\begin{array}{c}\text { Monitoring } \\
\text { wavelength } / \mathrm{nm}\end{array}$ & $\begin{array}{l}\text { Assessing } \\
\text { mode }\end{array}$ & $\begin{array}{c}\text { Power density } \\
/\left(\mathrm{W} \cdot \mathrm{cm}^{-2}\right)\end{array}$ & $\begin{array}{l}\text { Time range } \\
/ \mathrm{min}\end{array}$ & Ref. \\
\hline$\beta-\mathrm{NaYF}_{4}: \mathrm{Yb}^{3+}, \mathrm{Er}^{3+}$ & $\sim 27$ & $550 / 650$ & photostability & $5 \times 10^{6}$ & 60 & [24] \\
\hline $\mathrm{NaGdF}_{4}: \mathrm{Yb}^{3+}, \mathrm{Er}^{3+} @ \mathrm{NaGdF}_{4}$ & $\sim 40$ & $550 / 650$ & photostability & 150 & 240 & {$[23]$} \\
\hline $\mathrm{YVO}_{4}: \mathrm{Yb}^{3+}, \mathrm{Er}^{3+}$ & $\sim 39$ & $520 / 550 / 650$ & photostability & $8 \times 10^{3}$ & 8 & [41] \\
\hline $\mathrm{NaYF}_{4}: \mathrm{Yb}^{3+}, \mathrm{Ho}^{3+}, \mathrm{Tm}^{3+} @ \mathrm{NaYF}_{4}$ & $\sim 22$ & $450 / 475 / 545 / 645$ & photostability & $8 \times 10^{6}$ & 360 & [42] \\
\hline$\beta-\mathrm{NaYF}_{4}: \mathrm{Yb}^{3+} / \mathrm{Er}^{3+}$ & $\sim 10$ & $550 / 650$ & photostability & $10^{6}$ & 60 & [29] \\
\hline$\beta-\mathrm{NaGdF}_{4}: \mathrm{Yb}^{3+}, \mathrm{Er}^{3+} @ \mathrm{NaYF}_{4}$ & $\sim 20$ & $520 / 550 / 650$ & brightness & / & / & [43] \\
\hline $\begin{array}{c}\beta-\mathrm{NaGdF}_{4}: \mathrm{Yb}^{3+}, \mathrm{Er}^{3+} @ \mathrm{NaGdF}_{4} \\
@ \mathrm{SiO}_{2} @ \mathrm{NPTAT} \text {-doped } \mathrm{SiO}_{2}\end{array}$ & $\sim 39$ & 550 & & & & \\
\hline $\begin{array}{c}\beta-\mathrm{NaGdF}_{4}: \mathrm{Yb}^{3+}, \mathrm{Tm}^{3+} @ \mathrm{NaGdF}_{4} \\
@ \mathrm{SiO}_{2} @ \mathrm{NPTAT} \text {-doped } \mathrm{SiO}_{2}\end{array}$ & $\sim 38$ & 480 & photostability & / & 15 & {$[14]$} \\
\hline $\begin{array}{c}\mathrm{NaYbF}_{4}: \mathrm{Er}^{3+} @ \mathrm{NaYF}_{4} @ \mathrm{SiO}_{2} @ \text { rhodamine B } \\
\text { isothiocyanate-doped } \mathrm{SiO}_{2}\end{array}$ & $\sim 24$ & 650 & & & & \\
\hline
\end{tabular}


浓度。研究表明, 掺杂浓度过高时会导致体系荧光猝 灭。因此, 要得到高亮度的上转换发光纳米颗粒必须 对掺杂浓度进行优化。例如早期纳米颗粒群体性光学 性能研究显示, 低于 $100 \mathrm{~W} / \mathrm{cm}^{2}$ 激发时, 在掺杂了约 $20 \mathrm{~mol} \% \sim 40 \mathrm{~mol} \% \mathrm{Yb}^{3+}$ 的 $\mathrm{NaYF}_{4}$ 体系中, $\mathrm{Tm}^{3+}$ 的优化浓 度约在 $0.2 \mathrm{~mol} \% \sim 0.5 \mathrm{~mol} \%{ }^{[45-49]}$ 。但是, 最新的单颗粒 荧光研究结果表明, 在超高功率密度激发下, 该浓度亮度对应关系可以重新建立平衡机制 ${ }^{[9]}$ 。如图 4 所示, 对于 $\mathrm{Yb}^{3+}-\mathrm{Tm}^{3+}$ 组合, Jin 等报道在 $2.5 \times 10^{6} \mathrm{~W} / \mathrm{cm}^{2}$ 激发 下, $\mathrm{Tm}^{3+}$ 浓度可以高达 $8 \mathrm{~mol} \%$, 更重要的是高浓度高 强度激发下上转换苂光强度比例在增加。Gargas 等 ${ }^{[13]}$ 也在 $\mathrm{Yb}^{3+}-\mathrm{Er}^{3+}$ 组合中发现了该现象, $\mathrm{Er}^{3+}$ 浓度可以高 达 $20 \mathrm{~mol} \%$, 并通过苂光照片形象展示了随功率密度 增加到 $10^{6} \mathrm{~W} / \mathrm{cm}^{2}$ 量级时, 高掺颗粒的亮度超过低掺 颗粒(图 4(b))。上述结果均显示, 在单颗粒检测条件下, 激发功率密度相对较高, 此时上转换苂光强度和稀土 离子掺杂浓度之间的最优关系完全不同于群体性光 学检测条件下的。除整体上转换荧光强度以外, 对于 同一离子的不同能级之间的跃迁概率也发生大的变 化。我们发现常见的近红外至近红外上转换发光组合 $\left(20 \mathrm{Yb}^{3+}-2 \mathrm{Tm}^{3+}\right)$, 在单颗粒饱和激发下, 来自于 $\mathrm{Tm}^{3+}$ 的 4 光子蓝光发射要比其双光子近红外发射强约 70 倍 ${ }^{[30]}$, 如图 4(c)所示。

\section{3 偏振上转换发光}

单颗粒偏振光谱在半导体体系中吸引了众多研 究焦点, 而在稀土掺杂发光纳米材料中则很少见。 相对于半导体这类宽谱单带的发光中心, 稀土离子 掺杂上转换纳米颗粒的发光则呈现稀土离子 $4 \mathrm{f}-4 \mathrm{f}$ 跃迁的窄线宽多波段特性。这些特定的发光峰具有 光谱学指纹效应, 因此常被用于进行精确的理论分 析。如果这类发光呈现偏振依赖性, 将丰富发光学 科学内涵。尽管单个稀土离子的跃迁具有极化方向, 而当无数离子分布在同一体系中, 其离子跃迁是否 具有偏振依赖性成为一个值得探索的疑问。我们首 先对 $\mathrm{Tm}^{3+}-\mathrm{Yb}^{3+}$ 掺杂的 $\beta-\mathrm{NaYF}_{4}$ 微米棒进行上转换 发光偏振依赖特性的研究 ${ }^{[27]}$ 。如图 5 所示, 通过改 变发射偏振角度可获得偏振方向依赖的上转换荧光 图谱。在室温饱和激发条件下, 发现基于 $\mathrm{Tm}^{3+}:{ }^{1} \mathrm{D}_{2}$, ${ }^{1} \mathrm{G}_{4}$ 能级的晶体场䢃裂以及部分跃迁禁戒的单重态 至多重态跃迁 ${ }^{1} \mathrm{D}_{2} \rightarrow{ }^{3} \mathrm{~F}_{3},{ }^{1} \mathrm{G}_{4} \rightarrow{ }^{3} \mathrm{H}_{5}$ 的发光。进一步周 期性偏振依赖的荧光图谱检测可发现: 1$)^{1} \mathrm{G}_{4} \rightarrow{ }^{3} \mathrm{H}_{5}$, ${ }^{3} \mathrm{H}_{4} \rightarrow{ }^{3} \mathrm{H}_{6}$ 跃迁的偏振度接近 1, 即意味着在 $768 \mathrm{~nm}$ 和 $792 \mathrm{~nm}$ 波段实现了上转换线偏振光输出; 2$)^{1} \mathrm{G}_{4} \rightarrow$ ${ }^{3} \mathrm{H}_{5}$ 跃迁相对其它所有跃迁具有反周期性的偏振依 赖性。对比实验表明, 这些现象可能和离子跃迁偶
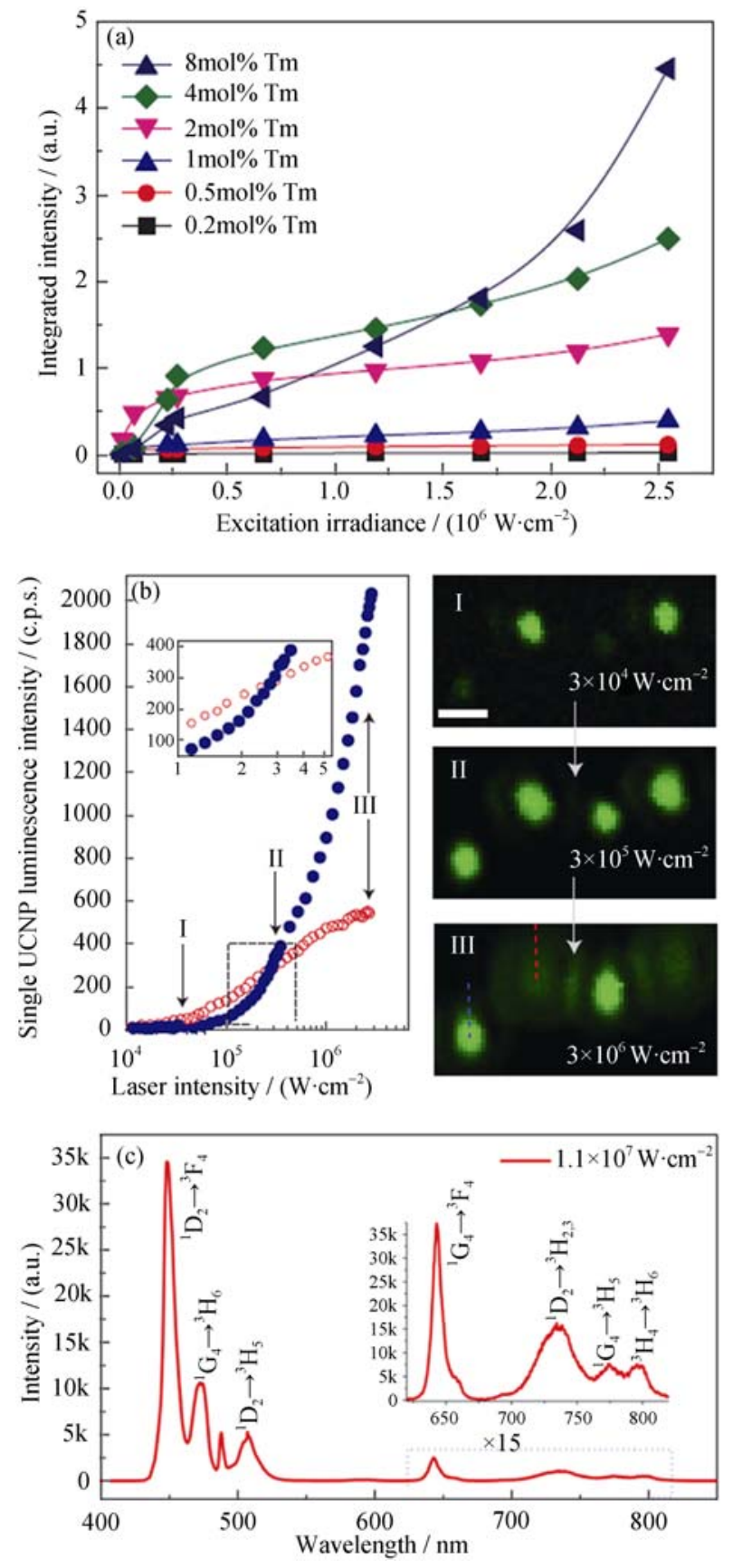

图 4 (a) 系列 $\mathrm{Tm}$ 掺杂纳米颗粒上转换发光积分强度随激发 功率变化关系; (b) 掺杂 $20 \% \mathrm{Yb}^{3+}-20 \% \mathrm{Er}^{3+}$ (蓝色) $/ 2 \% \mathrm{Er}^{3+}$ (红色) 的 $8 \mathrm{~nm}$ 单颗粒上转换强度随功率的变化关系。照片对应左图 I、II、III 功率下的共聚焦显微荧光图像; (c) 单个 $\beta-\mathrm{NaYF}_{4}$ : $20 \% \mathrm{Yb}^{3+}-2 \% \mathrm{Tm}^{3+}$ 纳米颗粒在 $1.1 \times 10^{7} \mathrm{~W} / \mathrm{cm}^{2} 980 \mathrm{~nm}$ 激光激 发下的是上转换苂光光谱图 ${ }^{[13,30,44]}$

Fig. 4 (a) Integrated upconrersion luminescence intensity ( $\sim 400$ $850 \mathrm{~nm}$ ) as a function of excitation irradiance for a series of $\mathrm{Tm}^{3+}$-doped nanoparticles. (b) Luminescence intensity of single $8 \mathrm{~nm}$ UCNPs with $20 \%$ (blue circles) and $2 \%$ (red circles) $\mathrm{Er}^{3+}$, each with $20 \% \mathrm{Yb}^{3+}$, plotted as a function of excitation intensity. Confocal luminescence images taken at points shown in (b) of single UCNPs containing a mixture of $2 \%$ and $20 \% \mathrm{Er}^{3+}$. (c) Emission spectra of single $\beta-\mathrm{NaYF}_{4}: 20 \% \mathrm{Yb}^{3+}-2 \% \mathrm{Tm}^{3+}$ nanoparticle excited with $980 \mathrm{~nm}$ laser illumination at the power density of $\sim 1.1 \times 10^{7} \mathrm{~W} / \mathrm{cm}^{2[13,30,44]}$ 

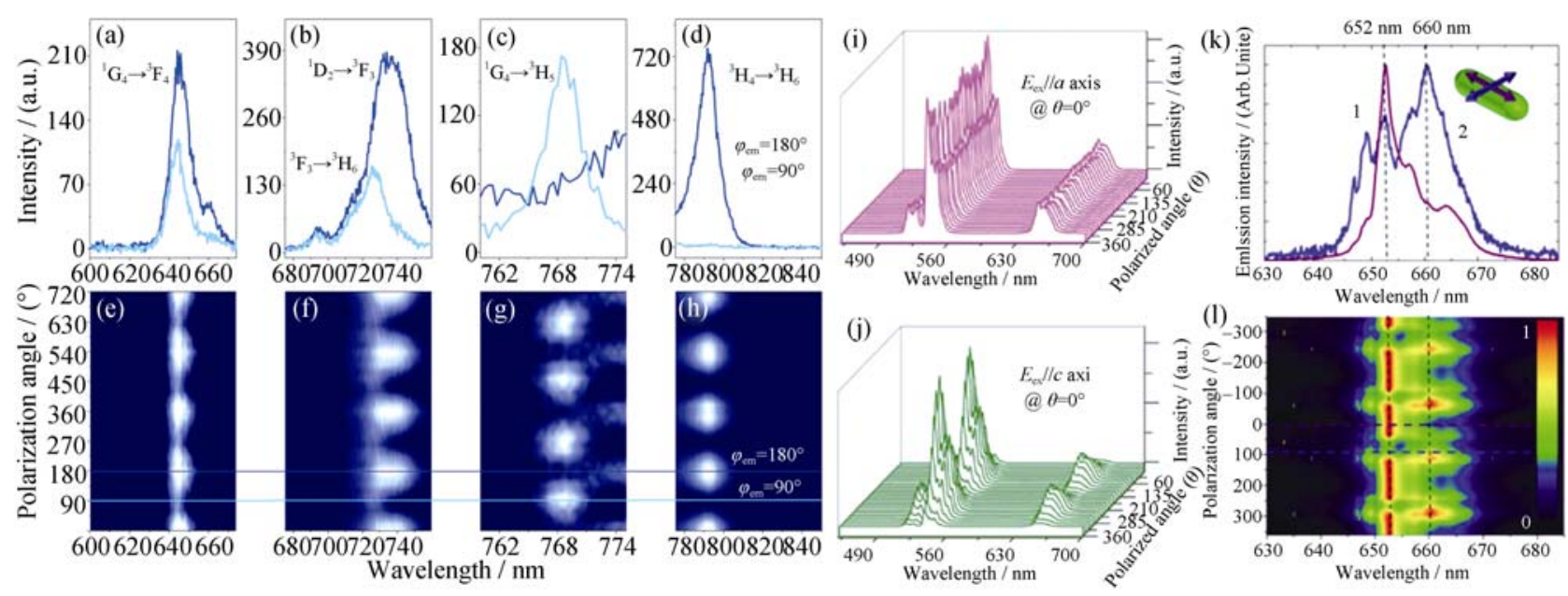

图 5 (a d)单根 $\beta-\mathrm{NaYF}_{4}$ : $\mathrm{Tm}^{3+}-\mathrm{Yb}^{3+}$ 微米棒的上转换发射光谱图, 分别对应 $\mathrm{Tm}^{3+}{ }^{3+}$ 跃迁: (a) ${ }^{1} \mathrm{G}_{4} \rightarrow{ }^{3} \mathrm{~F}_{4}$, (b) ${ }^{3} \mathrm{~F}_{3} \rightarrow{ }^{3} \mathrm{H}_{6},{ }^{1} \mathrm{D}_{2} \rightarrow{ }^{3} \mathrm{~F}_{3}$, (c) ${ }^{1} \mathrm{G}_{4} \rightarrow{ }^{3} \mathrm{H}_{5}$, $(d)^{3} \mathrm{H}_{4} \rightarrow{ }^{3} \mathrm{H}_{6} ;(\mathrm{e} \sim \mathrm{h})$ 各跃迁荧光强度随发射偏振角的变化关系; (i, j)单个纳米盘其晶体学 $\mathrm{a}$ 轴(i)或 $\mathrm{c}$ 轴(j)平行水平面时, 上转换发 射光谱随激发偏振角变化; $(\mathrm{k})$ 单根 $\mathrm{NaYF}_{4}$ : $\mathrm{Er}^{3+}, \mathrm{Yb}^{3+}$ 纳米棒其处于两个相垂直发射偏振角度时的发射光谱图。曲线 1 和曲线 2 表示发射偏振角平行和垂直纳米棒光轴。插图表示电场下两个不同偏振方向, 产生截然不同的光谱; (1)二维图谱表示红光波段的 发射强度随发射偏振角的变化关系 ${ }^{[27,31]}$

Fig. 5 (a-d) Emission spectra of UC from $\beta-\mathrm{NaYF}_{4}$ : $\mathrm{Tm}^{3+}-\mathrm{Yb}^{3+}$ single micro-rod in the transitions of $\mathrm{Tm}^{3+}$ : (a) ${ }^{1} \mathrm{G}_{4} \rightarrow{ }^{3} \mathrm{~F}_{4}$, (b) ${ }^{3} \mathrm{~F}_{3} \rightarrow{ }^{3} \mathrm{H}_{6}$, ${ }^{1} \mathrm{D}_{2} \rightarrow{ }^{3} \mathrm{~F}_{3}$, (c) ${ }^{1} \mathrm{G}_{4} \rightarrow{ }^{3} \mathrm{H}_{5}$, (d) ${ }^{3} \mathrm{H}_{4} \rightarrow{ }^{3} \mathrm{H}_{6}$, respectively. (e-h) The dependence of the corresponding spectra on emission polarization angle $\left(\varphi_{\mathrm{em}}\right)$. (i, j) UC luminescence spectra of a single nanodisk, whose $a$ axis or $c$ axis is parallel to horizontal plane, recorded at excitation polarization angles varying from $0^{\circ}$ to $360^{\circ}$, with no polarizer placed in the detection part. (k) Emission spectra from a single $\mathrm{NaYF}_{4}: \mathrm{Er}^{3+}, \mathrm{Yb}^{3+} \mathrm{UCNR}$ immobilized on a surface for two perpendicular emission polarization angles. Purple (blue) line represents recovered emission parallel (perpendicular) to the optical axis of the $\mathrm{NaYF}_{4}: \mathrm{Er}^{3+}, \mathrm{Yb}^{3+} \mathrm{UCNR}$. Inset represents these two different polarizations of the electric field that gives the two distinct spectra. (1) Two dimensional map represents emission intensity of red band as a function of emission polarization angle ${ }^{[27,31]}$

极矩取向及发光中心在晶体结构中所处的局域多面 体对称性相关。为了进一步从实验角度证实微米棒 上转换偏振各向异性的原因, 我们考虑光场偏振方 向和样品晶体学轴向的关系进行激发偏振检测 $[31]$ 。 结果显示, 当光场偏振方向和样品晶体学轴向平行 时, $\mathrm{Er}^{3+}:{ }^{2} \mathrm{H}_{11 / 2} \rightarrow{ }^{4} \mathrm{I}_{15 / 2},{ }^{4} \mathrm{~S}_{3 / 2} \rightarrow{ }^{4} \mathrm{I}_{15 / 2},{ }^{4} \mathrm{~F}_{9 / 2} \rightarrow{ }^{4} \mathrm{I}_{15 / 2}$ 跃迁 的偏振度分别达到 $0.78,0.79,0.74$, 而垂直时, 则偏 振依赖性基本消失。类似的, Haro-González 等 ${ }^{[36]}$ 采 用光镊法也证实了 $\mathrm{Er}^{3+}:{ }^{4} \mathrm{~F}_{9 / 2} \rightarrow{ }^{4} \mathrm{I}_{15 / 2}$ 红色上转换发光 具有明显的偏振依赖性。

\section{4 时间分辨成像}

寿命可以说是稀土离子电子跃迁除了光谱图像 以外的另一个重要指纹特征。通常, 稀土离子 $4 \mathrm{f}-4 \mathrm{f}$ 电子跃迁的寿命在微秒至毫秒量级。相对于其他长 寿命苂光材料, 稀土离子掺杂上转换纳米颗粒在传 感、生物成像及显示等领域更胜一筹。尽管上转换 发光的寿命可以通过掺杂离子的局域浓度进行调控, 可如果要针对特定应用进行一定范围内的精细调控 仍然是件困难的事。近期, $\mathrm{Lu}$ 等 ${ }^{[10]}$ 在 $\mathrm{NaYF}_{4}$ 纳米颗 粒所产生的 $475 \mathrm{~nm}$ 上转换荧光处实现了苂光寿命 从 25.6 662.4 $\mu \mathrm{s}$ 范围内调节的十几种粒子布居状 态。图 6 即为采用时间分辨技术共聚焦成像演示的
5 类不同寿命 $\mathrm{Tm}$ 掺杂上转换纳米颗粒，其中色彩为 虚拟显示。这是研究者首次采用瞬态荧光成像技术 而非常规的稳态光谱, 实现的单颗粒尺度表征。这 类上转换发光行为的多维度挖掘及展示有利于进一 步开拓上转换纳米颗粒更广阔和先端的应用前景。 譬如, 利用微纳尺度上转换苂光寿命的检测识别判 断微区内离子间能量传递 ${ }^{[50]}$ 。

\section{5 单颗粒上转换发光-等离子增强}

等离子体局域场作用由于其对荧光等的增强效 应而受到广泛关注，但也因其尚不明确的作用机理 以及猝灭并存现象而备受争议 ${ }^{[51-53]}$ 。其中公认的一个 关键因素是发光中心与等离子体之间的空间位置 ${ }^{[54]}$ 。 就这一点而言, 利用单颗粒检测手段, 通过上转换 纳米颗粒和金纳米球之间的距离调控, 研究者进行 了详尽的实验论证 ${ }^{[26]}$ 。如图 7(a)所示, 金属颗粒被 移动到上转换纳米颗粒附近, 并使两者中心轴和光 场偏振方向平行。因此, 从上转换发射光谱中(图 7(b))可以观察到 $\mathrm{Er}^{3+}$ 绿光和红光分别增强了 4.8 和 2.7 倍。该等离子体对上转换苂光增强效应还反映在 寿命曲线上升时间和衰减时间的缩短(图 7(c))。这种 基于单颗粒的空间位置准确控制型研究, 将为未来 杂化结构的优化设计提供重要指导意义。 

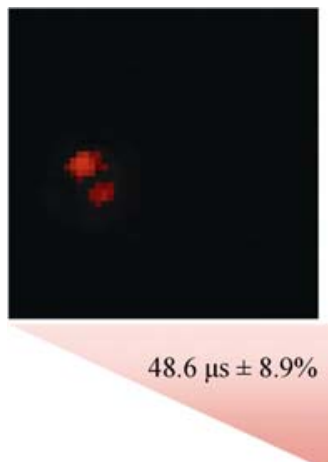

Lifetime ( $\mu s)$

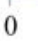

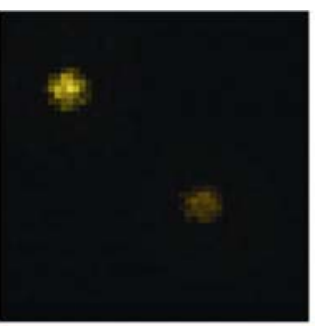

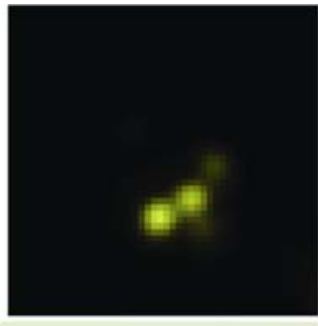

$126.5 \mu \mathrm{s} \pm 8.9 \%$
$182.6 \mu \mathrm{s} \pm 6.6 \%$

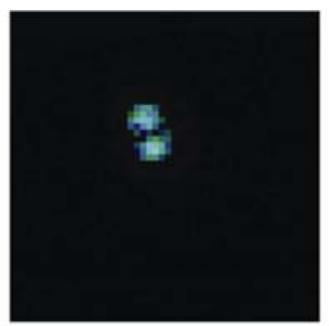

$501.1 \mu \mathrm{s} \pm 8.8 \%$

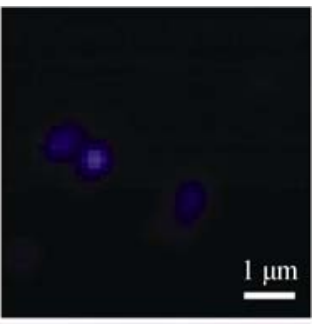

$668.8 \mu \mathrm{s} \pm 8.8 \%$

图 6 苂光寿命调节示范，即 $\mathrm{NaYF}_{4}: \mathrm{Yb}, \mathrm{Tm}$ 上转换纳米颗粒的时间分辨共聚焦图像 ${ }^{[10]}$

Fig. 6 Lifetime tuning scheme and time-resolved confocal images for $\mathrm{NaYF}_{4}$ : $\mathrm{Yb}$, $\mathrm{Tm}$ upconversion nanocrystals ${ }^{[10]}$

类似的, AFM 针尖增强也利用了等离子体局域 场效应，当金属针尖靠近物体时局域电场可以增强 发光中心激发或发射效率。近期, Mauser 等 ${ }^{[55}$ 报道了 $\mathrm{AFM}$ 扫描针尖对单个 $\mathrm{NaYF}_{4}: \mathrm{Yb}^{3+}-\mathrm{Er}^{3+}$ 纳米颗粒的 近场作用研究。极大的荧光增强结果(图 7(e))证实了
针尖近场增强效应，同时，通过计算可以发现增强 因子和近场远场实验构型下作用的对比结构基本一 致(图 7(d))。荧光寿命极剧缩短说明增强效应来自于 辐射速率加快(图 7(f))。这一研究显示了单颗粒光谱检 测技术在精密表征复杂上转换体系中的巨大前景。
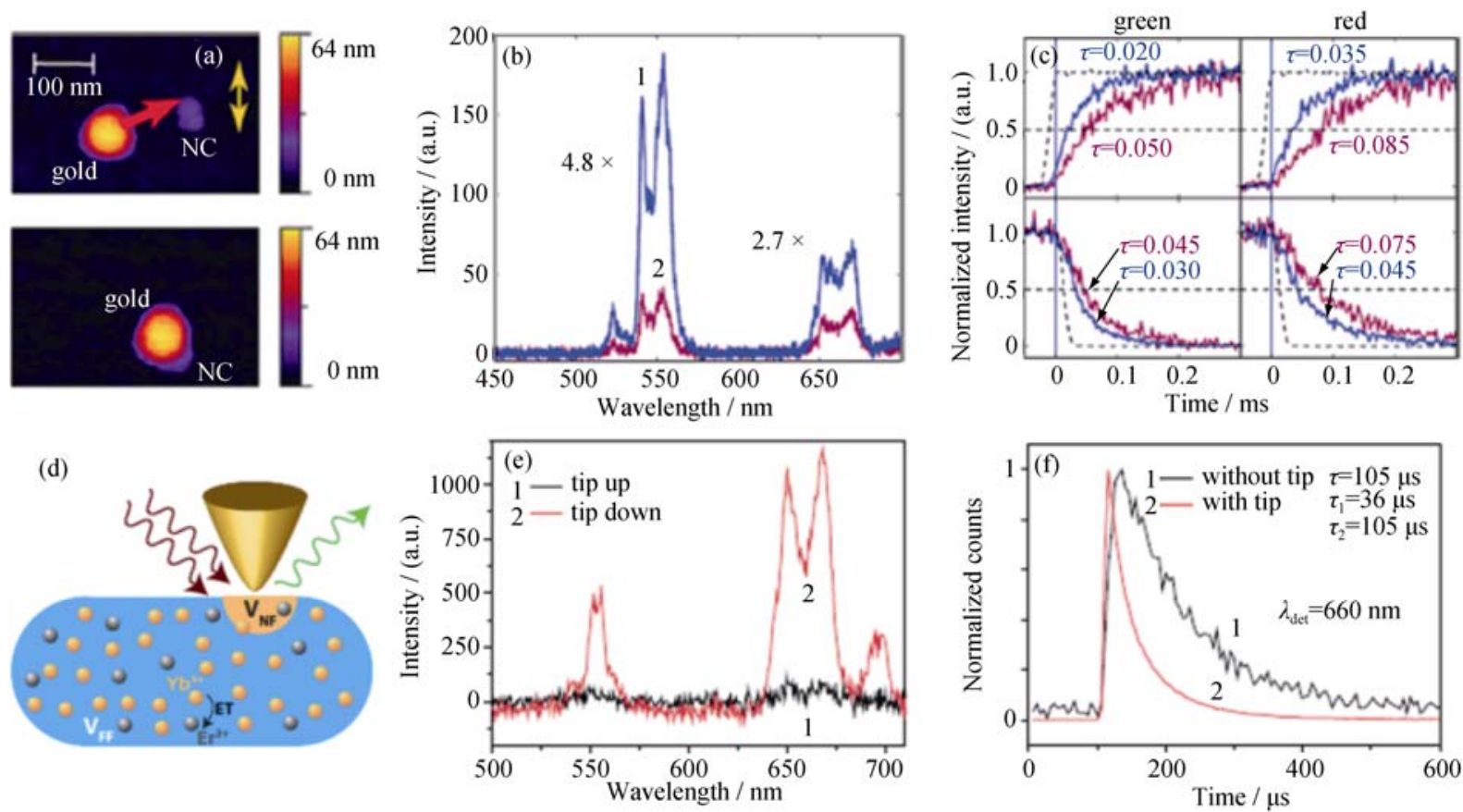

图 7 (a) AFM 图像展示纳米组装方式: $60 \mathrm{~nm}$ 金纳米球在 AFM 针尖拨动下靠近上转换纳米颗粒。黄色箭头表示激发光的偏振 方向; (b) 上转换纳米颗粒和金属小球靠近(曲线 1)、远离(曲线 2)时的发射光谱; (c) 上转换绿光(左侧)和红光(右侧)的上升(上侧) 及衰减(下侧)曲线; (d) 单个上转换纳米颗粒针尖增强效应示意图; (e) 针尖缩回和靠近时的上转换发射光谱; (f) $660 \mathrm{~nm}$ 处上转 换发光在针尖靠近和离开时的苂光衰减曲线 ${ }^{[26,55]}$

Fig. 7 (a) AFM image showing the nanoassembly approach: The $60 \mathrm{~nm}$ gold nanosphere is attached to the UCNPs with the help of the AFM tip. The yellow arrow indicates the polarization axis of the excitation light. (b) Upconversion emission spectra of the nanoparticle without (violet curve) and with (blue curve) the gold nanosphere in close vicinity. (c) Rise (upper) and decay times (lower) of the green (left) and red (right) emission with the color code as in part (b). (d) Schematic of the tip-enhancement of a single Upconversion nanoparticle. (e) Upconversion emission spectra with retracted and approached tip, respectively. (f) Decay curves for red emission detected with and without tip at $660 \mathrm{~nm}^{[26,55]}$ 


\section{3 结束语}

在过去几年中, 上转换纳米颗粒相关领域研究 主要集中在纳米制备、光物理学理论和一些生物、 能源、显示等方面的初步应用。面向更加广阔深入 的发展, 我们认为先进的技术推动力将十分必要, 这些技术主要致力于晶体生长动力学相关的本征结 构信息, 并结合考虑尺寸效应、表面效应和局域场 效应等对发光的影响。从这一层面来说, 对单个纳 米颗粒上转换发光行为的表征是一种突破对上转换 发光现有认识的极其有效的技术手段。它超越了普 遍采用的群体性光谱学检测方法, 具有众多优越性, 如展现纳米晶体个体差异甚至各向异性光谱特征、 探索新型光物理学现象等。

尽管近期越来越多的研究关注到单个纳米颗粒 的上转换发光行为, 但仍然需要更深入的思考以推 进这方面的研究达到显著的深度和广度。首先, 实 现单颗粒检测必须要克服的挑战是灵敏度问题。小 尺寸上转换纳米颗粒的荧光量子效率仍然很低, 目 前采用的检测依赖高达 $10^{6} \mathrm{~W} / \mathrm{cm}^{2}$ 量级的功率密度。 但对单颗粒应用而言, 过高的辐照光可能对单颗粒 所处的应用环境带来一些副作用甚至破坏性。此外, 正是由于受到若光效率的限制, 至今为止对单颗粒 的研究还只局限在效率相对较高的离子组合, 即 $\mathrm{Yb}^{3+}-\mathrm{Er}^{3+}, \mathrm{Yb}^{3+}-\mathrm{Tm}^{3+}$ 。因此, 从纳米制备层面需要考 虑开发更高品质的上转换纳米颗粒, 从光学技术层 面则可考虑定向激发或收集荧光等通过更有效的探 测来提高灵敏度。另一方面, 因为缺乏商用化的检 测设备, 只有少数课题组具备系统搭建和检测的能 力, 这客观限制了该领域的有效发展, 因此迫切需 要发展学科交叉性的研究团体。其次, 针对纳米尺 度的小颗粒, 需要开发更快速的纳米定位方法。以 共聚焦荧光扫描方法为例, 试样处理和逐点扫描来 定位单颗粒, 是非常耗时且依赖于经验。其三, 对于 单颗粒的高精度可控探测, 希望不仅仅局限于现象 学初步探究。例如在单颗粒尺度加入低温、压力、 磁场、电场等外场作用, 而使得上转换现象呈现更 强大的调制能力。单个纳米颗粒上转换发光还可能 在光学超分辨成像、生物诊疗动力学探测等领域将 具有重要应用前景。

\section{参考文献:}

[1] AUZEL F. Upconversion and anti-Stokes processes with $\mathrm{f}$ and $\mathrm{d}$ ions in solids. Chem. Rev. , 2004, 104(1): 139-173.

[2] WANG X, ZHUANG J, PENG Q, et al. A general strategy for nanocrystal synthesis. Nature, 2005, 437(7055): 121-124.

[3] WANG F, HAN Y, LIM C S, et al. Simultaneous phase and size control of upconversion nanocrystals through lanthanide doping. Nature, 2010, 463(7284): 1061-1065.

[4] ZHANG F, LI J, SHAN J, et al. Shape, size, and phase-controlled rare-earth fluoride nanocrystals with optical up-conversion properties. Chem-Eur. J., 2009, 15(41): 11010-11019.

[5] YI G S, CHOW G M. Synthesis of hexagonal-phase $\mathrm{NaYF}_{4}$ : Yb, Er and $\mathrm{NaYF}_{4}$ : $\mathrm{Yb}, \mathrm{Tm}$ nanocrystals with efficient up-conversion fluorescence. Adv. Funct. Mater., 2006, 16(18): 2324-2329.

[6] LIU D, XU X, DU Y, et al. Three-dimensional controlled growth of monodisperse sub-50 nm heterogeneous nanocrystals. Nat. Commun. , 2016, 7: 10254.

[7] ZHOU B, SHI B, JIN D, et al. Controlling upconversion nanocrystals for emerging applications. Nat. Nanotechnol., 2015, 10(11): 924-936.

[8] WANG F, DENG R, WANG J, et al. Tuning upconversion through energy migration in core-shell nanoparticles. Nat. Mater., 2011, 10(12): 968-973.

[9] ZHAO J B, JIN D Y, SCHARTNER E P, et al. Single-nanocrystal sensitivity achieved by enhanced upconversion luminescence. Nat. Nanotechnol., 2013, 8(10): 729-734.

[10] LU Y, ZHAO J, ZHANG R, et al. Tunable lifetime multiplexing using luminescent nanocrystals. Nat. Photonics, 2014, 8(1): 33-37.

[11] LU Y, LU J, ZHAO J, et al. On-the-fly decoding luminescence lifetimes in the microsecond region for lanthanide-encoded suspension arrays. Nat. Commun., 2014, 5: 3741.

[12] WANG J, DENG R, MACDONALD M A, et al. Enhancing multiphoton upconversion through energy clustering at sublattice level. Nat. Mater. , 2014, 13(2): 157-162.

[13] GARGAS D J, CHAN E M, OSTROWSKI A D, et al. Engineering bright sub-10-nm upconverting nanocrystals for single-molecule imaging. Nat. Nanotechnol., 2014, 9(4): 300-305.

[14] ZHOU L, WANG R, YAO C, et al. Single-band upconversion nanoprobes for multiplexed simultaneous in situ molecular mapping of cancer biomarkers. Nat. Commun., 2015, 6: 6938.

[15] FERNEE M J, TAMARAT P, LOUNIS B. Spectroscopy of single nanocrystals. Chem. Soc. Rev., 2014, 43(4): 1311-1337.

[16] SONNTAG M D, KLINGSPORN J M, ZRIMSEK A B, et al. Molecular plasmonics for nanoscale spectroscopy. Chem. Soc. Rev., 2014, 43(4): 1230-1247.

[17] CUI J, BEYLER A P, BISCHOF T S, et al. Deconstructing the photon stream from single nanocrystals: from binning to correlation. Chem. Soc. Rev., 2014, 43(4): 1287-1310.

[18] EMPEDOCLES S A, NEUHAUSER R, SHIMIZU K, et al. Photoluminescence from single semiconductor nanostructures. Adv. Mater., 1999, 11(15): 1243-1256.

[19] BLANTON S A, HINES M A, GUYOT-SIONNEST P. Photoluminescence wandering in single CdSe nanocrystals. Appl. Phys. Lett., 1996, 69(25): 3905-3907.

[20] NIRMAL M, DABBOUSI B O, BAWENDI M G, et al. Fluorescence intermittency in single cadmium selenide nanocrystals. $\mathrm{Na}$ ture, 1996, 383(6603): 802-804.

[21] EMPEDOCLES S, BAWENDI M. Quantum-confined stark effect in single CdSe nanocrystallite quantum dots. Science, 1997, 278(5346): 2114-2117.

[22] PARK Y I, LEE K T, SUH Y D, et al. Upconverting nanoparticles: a versatile platform for wide-field two-photon microscopy and multi-modal in vivo imaging. Chem. Soc. Rev., 2014, 44(6): 1302-1317.

[23] PARK Y I, KIM J H, LEE K T, et al. Nonblinking and nonbleaching upconverting nanoparticles as an optical imaging nanoprobe 
and T1 magnetic resonance imaging contrast agent. Adv. Mater., 2009, 21(44): 4467-4471.

[24] WU S W, HAN G, MILLIRON D J, et al. Non-blinking and photostable upconverted luminescence from single lanthanide- doped nanocrystals. Proc. Natl. Acad. Sci. USA, 2009, 106(27): 1091710921.

[25] SCHIETINGER S, MENEZES L D, LAURITZEN B, et al. Observation of size dependence in multicolor upconversion in single $\mathrm{Yb}^{3+}, \mathrm{Er}^{3+}$ codoped $\mathrm{NaYF}_{4}$ nanocrystals. Nano Lett., 2009, 9(6): 2477-2481.

[26] SCHIETINGER S, AICHELE T, WANG H Q, et al. Plasmon-enhanced upconversion in single $\mathrm{NaYF}_{4}: \mathrm{Yb}^{3+} / \mathrm{Er}^{3+}$ codoped nanocrystals. Nano Lett., 2010, 10(1): 134-138.

[27] ZHOU J J, CHEN G X, WU E, et al. Ultrasensitive polarized up-conversion of $\mathrm{Tm}^{3+}-\mathrm{Yb}^{3+}$ doped beta- $\mathrm{NaYF}_{4}$ single nanorod. Nano Lett., 2013, 13(5): 2241-2246.

[28] KOLESOV R, XIA K, REUTER R, et al. Optical detection of a single rare-earth ion in a crystal. Nat. Commun., 2012, 3: 1029.

[29] OSTROWSKI A D, CHAN E M, GARGAS D J, et al. Controlled synthesis and single-particle imaging of bright, sub-10 nm lanthanide-doped upconverting nanocrystals. ACS Nano, 2012, 6(3): 2686-2692.

[30] ZHOU J J, CHEN G X, ZHU Y B, et al. Intense multiphoton upconversion of $\mathrm{Yb}^{3+}-\mathrm{Tm}^{3+}$ doped beta- $\mathrm{NaYF}_{4}$ individual nanocrystals by saturation excitation. J. Mater. Chem. C, 2015, 3(2): 364-369.

[31] CHEN P, SONG M, WU E, et al. Polarization modulated upconversion luminescence: single particle vs. few-particle aggregates. Nanoscale, 2015, 7(15): 6462-6466.

[32] SCHARTNER E P, JIN D Y, EBENDORFF-HEIDEPRIEM H, et al. Lanthanide upconversion within microstructured optical fibers: improved detection limits for sensing and the demonstration of a new tool for nanocrystal characterization. Nanoscale, 2012, 4(23): 7448-7451.

[33] SCHARTNER E P, JIN D, EBENDORFF-HEIDEPRIEM H, et al. Lanthanide upconversion nanocrystals within microstructured optical fibres; a sensitive platform for biosensing and a new tool for nanocrystal characterisation. Third Asia Pacific Optical Sensors Conference, 2012, 8351.

[34] SCHARTNER E P, JIN D Y, ZHAO J B, et al. Sensitive Detection of $\mathrm{NaYF}_{4}: \mathrm{Yb} / \mathrm{Tm}$ Nanoparticles Using Suspended Core Microstructured Optical Fibers. Colloidal Nanocrystals for Biomedical Applications Viii, 2013: 8595.

[35] RODRIGUEZ-SEVILLA P, RODRIGUEZ-RODRIGUEZ H, PEDRONI M, et al. Assessing single upconverting nanoparticle luminescence by optical tweezers. Nano Lett. , 2015, 15(8): 5068- 5074.

[36] RODRIGUEZ-SEVILLA P, LABRADOR-PAEZ L, WAWRZYN CZYK D, et al. Determining the 3D orientation of optically trapped upconverting nanorods by in situ single-particle polarized spectroscopy. Nanoscale, 2015, 8(1): 300-308.

[37] DICKSON R M, CUBITT A B, TSIEN R Y, et al. On/off blinking and switching behaviour of single molecules of green fluorescent protein. Nature, 1997, 388(6640): 355-358.

[38] NIRMAL M, DABBOUSI B, BAWENDI M, et al. Fluorescence intermittency in single cadmium selenide nanocrystals. Nature, 1996, 383(6603): 802-804.
[39] GALLAND C, GHOSH Y, STEINBRUCK A, et al. Two types of luminescence blinking revealed by spectroelectrochemistry of single quantum dots. Nature, 2011, 479(7372): 203-207.

[40] BARNES M, MEHTA A, THUNDAT T, et al. On-off blinking and multiple bright states of single europium ions in $\mathrm{Eu}^{3+}: \mathrm{Y}_{2} \mathrm{O}_{3}$ nanocrystals. J. Phys. Chem. B, 2000, 104(26): 6099-6102.

[41] MIALON G, TURKCAN S, DANTELlE G, et al. High up-conversion efficiency of $\mathrm{YVO}_{4}: \mathrm{Yb}$, Er nanoparticles in water down to the single-particle level. J. Phys. Chem. C, 2010, 114(51): 22449-22454.

[42] ZHANG F, SHI Q, ZHANG Y, et al. Fluorescence upconversion microbarcodes for multiplexed biological detection: nucleic acid encoding. Adv. Mater., 2011, 23(33): 3775-3779.

[43] LI X, WANG R, ZHANG F, et al. Engineering homogeneous doping in single nanoparticle to enhance upconversion efficiency. $\mathrm{Na}$ no Lett., 2014, 14(6): 3634-3639.

[44] ZHAO J, JIN D, SCHARTNER E P, et al. Single-nanocrystal sensitivity achieved by enhanced upconversion luminescence. Nat. Nanotechnol., 2013, 8(10): 729-734.

[45] ZHANG H, LI Y, LIN Y, et al. Composition tuning the upconversion emission in $\mathrm{NaYF}_{4}: \mathrm{Yb} / \mathrm{Tm}$ hexaplate nanocrystals. Nanoscale, 2011, 3(3): 963-966.

[46] YIN A X, ZHANG Y W, SUN L D, et al. Colloidal synthesis and blue based multicolor upconversion emissions of size and composition controlled monodisperse hexagonal $\mathrm{NaYF}_{4}: \mathrm{Yb}, \mathrm{Tm}$ nanocrystals. Nanoscale, 2010, 2(6): 953-959.

[47] MAHALINGAM V, VETRONE F, NACCACHE R, et al. Colloidal $\mathrm{Tm}^{3+} / \mathrm{Yb}^{3+}$-doped $\mathrm{LiYF}_{4}$ nanocrystals: multiple luminescence spanning the UV to NIR regions via low-energy excitation. Adv. Mater., 2009, 21(40): 4025-4028.

[48] KR MER K W, BINER D, FREI G, et al. Hexagonal sodium yttrium fluoride based green and blue emitting upconversion phosphors. Chemistry of Materials, 2004, 16(7): 1244-1251.

[49] LIANG L, WU H, HU H, et al. Enhanced blue and green upconversion in hydrothermally synthesized hexagonal $\mathrm{NaY}_{1-x} \mathrm{Yb}_{x} \mathrm{~F}_{4}$ : $\mathrm{Ln}^{3+}\left(\mathrm{Ln}^{3+}=\mathrm{Er}^{3+}\right.$ or $\left.\mathrm{Tm}^{3+}\right)$. J. Alloys Compd., 2004, 368(1): 94-100.

[50] ZHANG Y H, ZHANG L X, DENG R R, et al. Multicolor barcoding in a single upconversion crystal. J. Am. Chem. Soc., 2014, 136(13): 4893-4896.

[51] GLASS A M, LIAO P F, BERGMAN J G, et al. Interaction of metal particles with adsorbed dye molecules: absorption and luminescence. Opt. Lett., 1980, 5(9): 368-370.

[52] LAKOWICZ J R. Radiative decay engineering: biophysical and biomedical applications. Anal. Biochem., 2001, 298(1): 1-24.

[53] DULKEITH E, MORTEANI A C, NIEDEREICHHOLZ T, et al. Fluorescence quenching of dye molecules near gold nanoparticles: radiative and nonradiative effects. Phys. Rev. Lett., 2002, 89(20): 203002.

[54] SABOKTAKIN M, YE X, OH S J, et al. Metal-enhanced upconversion luminescence tunable through metal nanoparticle-nanophosphor separation. ACS Nano, 2012, 6(10): 8758-8766.

[55] MAUSER N, PIATKOWSKI D, MANCABELLI T, et al. Tip-enhancement of up-conversion photoluminescence from rareearth ion doped nanocrystals. ACS Nano, 2015, 9(4): 3617-3626. 